\title{
Stroke as a first sign of a malignant cancer (a case of thrombotic non infectious endocarditis)
}

\begin{abstract}
Nonbacterial thrombotic endocarditis is a disease which is characterized by abnormal deposit of fibrin and platelets over heart valves. It can appear in relation with disseminated cancer, connective tissue diseases or severe infections. Is not common as a first sign of an occult cancer and that is why we present our clinical case based on a patient who suffered from stroke as the first symptom of lung cancer.
\end{abstract}

Keywords: nonbacterial endocarditis, stroke, cancer
Volume 6 Issue I - 2017

\author{
Alba Velázquez Benito, Alejandro Viloria \\ Alebesque, Ana Suller Martí, Cristina Pérez \\ Lázaro, Carlos Tejero Juste, Sonia Santos \\ Lasaosa \\ Neurology Department, Hospital Clínico Universitario Lozano \\ Blesa, Spain
}

Correspondence: Alba Velázquez Benito, Neurology Department, Hospital Clínico Universitario Lozano Blesa, Zaragoza, Spain, Tel 0034 686913513,

Email albavelazquez83@gmail.com

Received: October 3I, 20I6 | Published: January 27, 2017

\section{Case report}

Nonbacterial thrombotic endocarditis is a rare disease that is commonly related to many other diseases such as disseminated cancer. It can produce embolism to many organs, such as lungs and brain. In the other hand, is not common to find brain stroke as the first sign of a malignant neoplasia due to a nonbacterial thrombotic endocarditis. Because of that, we think this case could be interesting.

We present a 69-years-old male, right handed and non-smoker, with medical history of diabetes without insulin treatment, dyslipidemia and deep vein thrombosis many years ago. Regular treatment: atorvastatin and metformin.

He went to the emergency room after having a left arm paresis followed by dysarthria. These symptoms lasted for less than one hour with complete recovering after that, because of that the patient was admitted with the diagnosis of transient ischemic attack. At the moment of admission, patient had normal arterial pressure levels, normal heart rate and no fever. The complementary exams that were done in the ER were unremarkable including a CT brain scan which did not show anything abnormal at the moment.

After 48hours of EKG monitoring, the patient suffered sudden loss of consciousness. When he recovered it spontaneously, his vital signs remained normal and his neurological exam showed left hemiparesis, severe dysarthria, left hemianopsia, left Babinski sign and loss of sensitivity in left body, with a total of 18 points in the National Health Institute Scale (NIHSS). An urgent CT brain scan showed a hypodense lesion regarding right middle cerebral artery, suggesting acute stroke. According to these findings, acute treatment with Alteplase was not administered.

In the next hours, patient got progressively better so he only presented mild left hemiparesis and mild dysarthria. After 48hours, he presented again loss of consciousness followed by worsened dysarthria and nausea. Another CT scan was done, with a new hypodense lesion in left cerebellar hemisphere Figure 1. At that moment, neurological exam showed drowsiness, severe left hemiparesis, loss of sensitivity in left body, left Babinski sign, severe dysarthria and dysphagia.

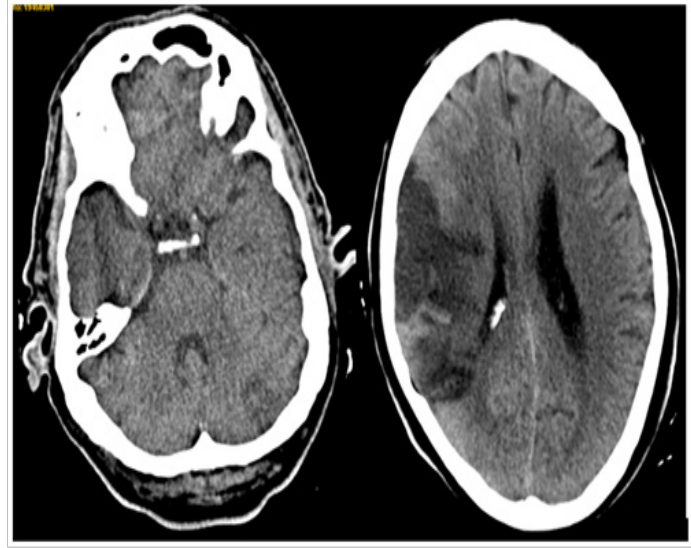

Figure I Brain CT scan without contrast, showing right middle cerebral artery stroke and left cerebellar hypodense lesion.

General exam showed normal cardiac rhythm with some roncus in left lung and digital clubbing affecting both hands that have not been noticed before (patient's wife confirmed they had appeared recently). She also told the patient had lost almost 6kg in the last months.

With a diagnosis of brain stroke regarding different vascular regions, the suspected etiology was endocarditis. Blood tests showed increased glomerular sedimentation speed, increased D-dimer (50417) and low platelets (115000). Other complementary exams such as blood serologies, blood and urine cultures were negative. Neurosonological study showed carotid atheromatosis without significant stenosis and a right middle cerebral artery occlusion. In the transthoracic echocardiography a lesion over mitral valvular veil was seen (Figure 2).

Furthermore, a chest CT scan was performed, showing a lung lesion in the low left lobe including left bronchus, lung lymphadenopathy, left pleura metastatis, left kidney stroke and bilateral suprarenal metastasis. A brain MRI confirmed right middle cerebral artery stroke, left posterior- inferior cerebellar artery stroke and right anterior cerebral artery stroke (Figure 3). 


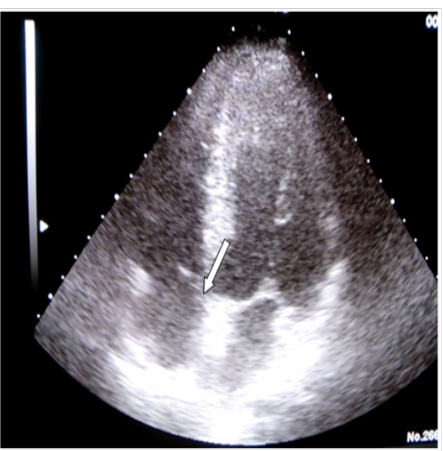

Figure 2 Vegetation over mitral vail, showed with transthoracic echocardiography.

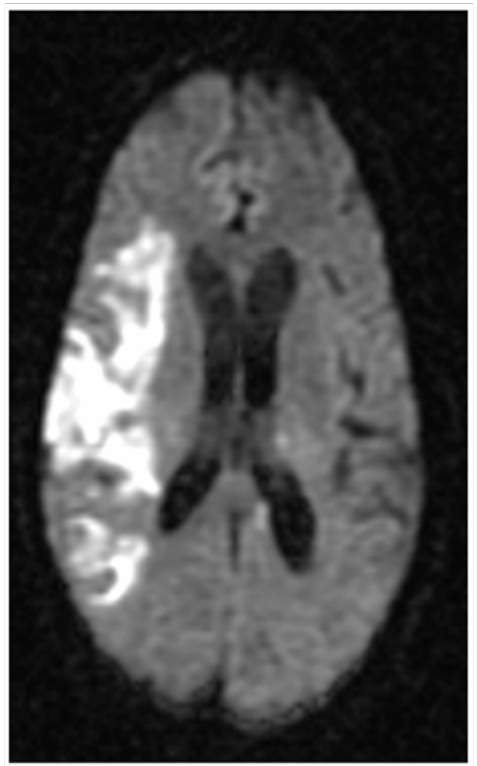

Figure 3 Brain MRI, difussion-weighted images showing right middle cerebral artery stroke.

A final diagnosis of nonbacterial thrombotic endocarditis was done and treatment with low weight heparin was started. Anyway, the patient worsened fastly and died several weeks after the diagnosis was done.

Nonbacterial thrombotic endocarditis have a prevalence of $0.3 \%$ 9.3\% regarding some necropsies ${ }^{1}$ series that have been published. It is commonly related to disseminated cancer, severe infections, connective tissue diseases or immunodeficiency.

Due to its low prevalence, the final diagnosis usually comes late and this is even more difficult when it happens in a patient with unknown cancer such as our clinical case

We must suspect a nonbacterial thrombotic endocarditis in a patient with different embolisms to different organs and unknown embolic causes. The organ that is affected the most is the brain ${ }^{2-4}$ followed by lungs, kidneys and heart although brain stroke is an uncommon first sign of a malignant neoplasia.

It is more common to find bilateral stroke, specially in middle cerebral arteries (posterior arteries are not usually affected). Non bacterial endocarditis is caused because of abnormal fibrin and platelets deposits ${ }^{5}$ in heart valvs that could be previously injured or not. Heart vegetations are commonly located in mitral or aortic valvs and they could happen at the same time. Some genetic predisposition, such as an anormal thrombophilic state have been suggested. ${ }^{6,7}$

The diagnosis must be done with echocardiography, ${ }^{8}$ specially with tranesophagic one. When brain stroke is suspected a CT scan is highly recommended although MRI could be more useful when the diagnosis is uncertain. Recommended treatment is anticoagulation, mainly with low-molecular weight heparin and cardiac surgery will only be required if the patient develops a cardiac failure and the underlying cancer have good outcome.

Finally, we emphasize that nonbacterial thrombotic endocarditis is a severe disease that is underdiagnosed and usually affects patients with disseminated cancer that could be unknown at the time that symptoms appear. ${ }^{10,11}$ Sometimes its diagnosis could be really difficult specially when the underlying diseases (cancer, connective tissue, immunodeficiency) is unknown.

\section{Acknowledgements}

None.

\section{Conflict of interest}

The author declares no conflict of interest.

\section{References}

1. Llenas-García J. et Endocarditis trombótica no bacteriana: estudio clínico-patológico de uma serie necrópsica. Ver Esp Cardiol. 2007;60(5):493-500.

2. Singhal AB, Topcuoglu MA, Buoanno FS. Acute ischemic stroke patterns in infective and nonbacterial thrombotic endocarditis. A diffusion-weighted thrombotic magnetic resonance imaging study. Stroke. 2002;33(5):1267-1273

3. Borowoski A, Ghdosizad A, Cohnen M, et al. Recurrent embolism in the course of marantic endocarditis. Ann Thorac Surg. 2005;79(6):21452147.

4. Taccone FS, Jeangette SM, Blecic SA. First-ever stroke as initial presentation of systemic cancer. J Stroke Cerebrovasc Dis. 2008;17(4):169 174.

5. El-Shami K, Griffiths E, Streiff M. Nonbacterial thrombotic endocarditis in cancer patients: pathogenesis, diagnosis and treatment. The oncologist 2007; $12: 518-523$.

6. Durante-Mangoni E, Iossa D, Nappi F, et al. Inherited hyper-homocysteinemia as a cause of nonbacterial thrombotic endocarditis. J Heart Valve Dis. 2011;20(2):232-233

7. Müller H, Renner M, Helmke BM, et al. Deleted in Malignant Brain Tumors 1 is up-regulated in bacterial endocarditis and binds to components of vegetations. J Thorac Cardiovasc Surg. 2009;138(3):725-732.

8. Roldan CA, Qualls CR, Sibbit JR. Transthoracic versus transesophageal echocardiography for detection of Libman-Sacks endocarditis:a randomized controlled study. J Rheumatol. 2008;35(2):224-229.

9. Kanthasamy V, Natarajan I. Rare and challenging case of stroke as a manifestation of non bacterial thrombotic endocarditis in an underlying ovarian clear cell carcinoma. JRSM Open. 2016;7(11):2054270416669304.

10. Higes-Pascual F, Tello-Blasco S, Fernández-Santos A, et al. Isquemia cerebral secundaria a una endocarditis trombótica no bacteriana como forma de presentación deun tumor ovárico. Rev Neurol. 2005;41(7):404408

11. Fanale MA, Zeldenrust SR, Moynihan TJ. Some unusual complications of malignancies. J Clin Oncol. 2002;19:4111-4114. 